\title{
Profile of clindamycin phosphate I.2\%/benzoyl peroxide $3.75 \%$ aqueous gel for the treatment of acne vulgaris
}

This article was published in the following Dove Press journal:

Clinical, Cosmetic and Investigational Dermatology

29 October 2015

Number of times this article has been viewed

\author{
Tuyet A Nguyen ${ }^{1,2}$ \\ Lawrence F Eichenfield ${ }^{1,3}$ \\ 'Division of Pediatric and Adolescent \\ Dermatology, Rady Children's \\ Hospital, San Diego, CA, ${ }^{2}$ Department \\ of Medicine, Albert Einstein College \\ of Medicine, Bronx, NY, ${ }^{3}$ Department \\ of Dermatology, University of \\ California, San Diego, La Jolla, CA, \\ USA
}

\begin{abstract}
Acne vulgaris is a common and chronic skin disease, and is a frequent source of morbidity for affected patients. Treatment of acne vulgaris is often difficult due to the multifactorial nature of this disease. Combination therapy, such as that containing clindamycin and benzoyl peroxide, has become the standard of care. Several fixed formulations of clindamycin $1 \%$ and benzoyl peroxide of varying concentrations are available and have been used with considerable success. The major limitation is irritation and dryness from higher concentrations of benzoyl peroxide, and a combination providing optimal efficacy and tolerability has yet to be determined. Recently, a clindamycin and benzoyl peroxide $3.75 \%$ fixed combination formulation was developed. Studies have suggested that this formulation may be a safe and effective treatment regimen for patients with acne vulgaris. Here, we provide a brief review of acne pathogenesis, benzoyl peroxide and clindamycin, and profile a new Clindamycin-BP 3.75\% fixed combination gel for the treatment of moderate-to-severe acne vulgaris.
\end{abstract}

Keywords: acne vulgaris, benzoyl peroxide, clindamycin

\section{Introduction}

Acne vulgaris is a common and chronic skin disease affecting millions of people worldwide. It is the most common skin disorder in the USA and is the number one reason for visiting a dermatologist. ${ }^{1}$ It is a common source of morbidity, and a variety of topical and systemic medications have been developed to treat acne. However, because of the multifactorial nature of acne, effective treatment is often difficult to obtain. Combination therapies targeting different aspects of acne pathogenesis have become the standard of care. ${ }^{2}$ Treatment with topical clindamycin in combination with benzoyl peroxide (BP) has been found to be superior to either medication alone. ${ }^{3}$ This combination appears to target both inflammatory and noninflammatory acne, and as such, has become a major treatment option for patients with acne. Several fixed formulations of clindamycin 1\% and BP (varying concentrations) have been used with considerable success. ${ }^{4-7}$ The major limitation associated with many fixed combinations is irritation and dryness from higher concentrations of BP. ${ }^{8}$ Here, we will provide a brief overview of acne pathogenesis, background on the use of BP and clindamycin, and profile a new clindamycin-BP 3.75\% fixed combination gel for the treatment of moderate-to-severe acne vulgaris.

\section{Background on acne vulgaris}

Acne vulgaris is the most common cutaneous disorder worldwide. It is particularly common in adolescents and young adults and is often a significant source of anxiety, 
embarrassment, and psychosocial morbidity for affected patients. ${ }^{9}$ Although most cases of acne vulgaris are selflimited or responsive to topical medications alone, severe cases may last well into adulthood and may be associated with scarring, persistent hyperpigmentation, and significant psychosocial consequences. ${ }^{2}$ Clinically, acne is characterized by a combination of noninflammatory open and closed comedones and inflammatory papules and pustules, typically affecting areas with a high proportion of sebaceous follicles, such as the face, chest, and back.

The pathogenesis of acne vulgaris is a multifactorial process, involving follicular hyperkeratinization and obstruction, increased sebum production, Propionibacterium acnes colonization, and inflammation. ${ }^{10}$ One of the major factors in the early formation of acne appears to be sebaceous follicle obstruction caused by follicular hyperkeratinization and deposition of keratinocytes in the follicle orifice leading to microcomedones. ${ }^{10,11}$ The cause and pathogenesis of follicular hyperkeratinization is still not completely understood. It is thought to be associated with changing hormonal influences during puberty. Higher levels of dihydrotestosterone have been found to act on infundibular keratinocytes, contributing to the hyperkeratinzation seen in acne pathogenesis. ${ }^{12}$ Additionally, increased expression of IL-1 $\alpha, \mathrm{K} 16$, and filaggrin has been found in conjunction with follicular hyperkeratinization, and they are thought to be contributing factors. ${ }^{13,14}$ It is still unclear why follicular hyperkeratinization and increased keratinocyte proliferation occur in some individuals, but not others.

Increased sebum production during puberty is also a major contributing factor in the pathogenesis of acne vulgaris. During puberty, androgens play an important role in altering the sebaceous unit expression in the skin. Sebaceous units have been found to contain enzymes that are capable of synthesizing androgens from precursor molecules. ${ }^{12}$ Additionally, androgens can have a stimulatory effect on sebocyte proliferation..$^{15}$ Interestingly, increased levels of lipoperoxides and decreased levels of vitamin $\mathrm{E}$ have been found in the sebum of acne patients, indicating that there is not only excessive sebum production in acne patients, but also altered sebum composition. ${ }^{16}$ Therefore, sebaceous gland growth, increased sebum excretion, and altered sebum composition likely predispose certain patients toward acne pathogenesis.

Follicular obstruction caused by hyperkeratinization in conjunction with increased sebum production during puberty provides an excellent environment for bacterial growth, particularly P. acnes. P. acnes is an anaerobic, Gram-positive bacteria that is typically present in normal skin flora, but can grow rapidly in areas of obstruction or increased sebum production. P. acnes has been found to be highly associated with acne and has been found to induce proinflammatory and antimicrobial factors, leading to the characteristic inflammation seen in acne vulgaris. ${ }^{17,18}$ Because $P$. acnes is part of the body's natural flora, it is likely that only certain strains with the ability to induce changes in immune response predispose patients to acne vulgaris.

Another important feature in the pathogenesis of acne vulgaris is inflammation. The presence of increased inflammatory cytokines is common to all patients with acne vulgaris. Inflammation is believed to play an important role in acne progression and increases the risk of scarring. ${ }^{19}$ Follicular hyperkertinization, abnormal sebum properties, and the presence of $P$. acnes all appear to contribute to the production of an inflammatory response in acne patients. ${ }^{17}$ Alterations in the immune response and the increased presence of inflammatory mediators in acne patients suggest that acne is an inflammatory skin disorder, and targeting the inflammatory response may improve acne symptoms.

\section{Benzoyl peroxide}

BP is often a mainstay in acne treatment regimens today. BP has known bactericidal properties. ${ }^{20}$ An in vitro study by Waller et $\mathrm{a}^{21}$ demonstrated that the minimum inhibitory concentrations against $P$. acnes was $128 \mu \mathrm{g} / \mathrm{mL}$, making it an effective agent for targeting $P$. acnes in the pathogenesis of acne vulgaris. The second mechanism of action by which BP targets acne is through its keratolytic effects. BP has been found to be more effective in disrupting stratum corneum cohesion compared to salicylic acid, retinoic acid, and vehicle. ${ }^{21}$ Additionally, it also has been found to have mild anti-inflammatory properties..$^{22}$ Interestingly, bacterial resistance to BP has not been reported, and use of BP in combination with topical antibiotics may even reduce the risk of $P$. acnes resistance to these agents as well., ${ }^{2,23,24}$ Although BP can be associated with some dryness and irritation, and occasional allergic contact dermatitis, it is generally a well-tolerated, safe, and effective topical medication for the treatment of acne vulgaris. ${ }^{24}$

\section{Topical antibiotics}

The most common topical antibiotics used for the treatment of acne include clindamycin and erythromycin. These antibiotics are used for acne treatment because they target P. acnes colonization and proliferation. ${ }^{25}$ Clindamycin and erythromycin are antibiotics that work by targeting the 50s subunit of bacterial ribosomes and interfering with protein synthesis, thereby exerting antibacterial effects. In 
addition to antibacterial activities, clindamycin also has anti-inflammatory properties. It is well known that $P$. acnes can induce an inflammatory response. Clindamycin has been shown to inhibit this $P$. acnes-driven inflammatory response as well as inhibit the expression of proinflammatory cytokines, such as IL-1 $\beta$, IL- 6 , INF- $\gamma$, TNF- $\alpha$, and GM-CSF. ${ }^{26}$ Additionally, studies have demonstrated that clindamycin use can also lead to increased phagocytosis and opsonization and an improved inflammatory response. ${ }^{26}$ Although these topical antibiotics have been shown to display considerable success in the treatment of acne vulgaris, they are rarely used as a monotherapy because of the risk for antimicrobial resistance. ${ }^{25,27}$

\section{Combination therapy: benzoyl peroxide and topical antibiotics}

Although topical antibiotics have been shown to be efficacious in the treatment of acne vulgaris, a major limitation for their use as monotherapy is the associated risk of antimicrobial resistance. ${ }^{25,27}$ Before the introduction of combination therapy, the emergence of erythromycin-resistant and clindamycin-resistant $P$. acnes in patients receiving topical or systemic antibiotics alone was a significant problem. ${ }^{28}$ Therefore, combination therapy utilizing at least two medications is currently the gold standard for treatment of acne vulgaris because it targets more than one factor in acne pathogenesis and prevents antimicrobial resistance. ${ }^{2}$ Topical combination therapy can involve the use of several medications together, such as BP, antibiotics, and retinoids, or may be available as fixed-dose combination therapies.

\section{Benzoyl peroxide and erythromycin}

The first studies combining BP with a topical antibiotic were performed using erythromycin rather than clindamycin. In 1983, Chalker et $\mathrm{al}^{29}$ sought to determine the efficacy of a combination erythromycin 3\% and BP 5\% gel in comparison to either medication alone and vehicle. They found that the combination of erythromycin and BP therapy had superior efficacy to vehicle and to either medication alone. There was also a significant improvement in mean pustule, papule, and inflammatory lesion count in the combination therapy group compared to vehicle, BP alone, or erythromycin alone. ${ }^{29}$ Eady et $\mathrm{a}^{28}$ also confirmed that combination of $\mathrm{BP}$ and erythromycin is superior to either product alone, and a checkerboard titration of BP and erythromycin at varying concentrations was used to determine whether this finding was due to synergistic antibacterial effects. They concluded that the improved efficacy of this combination therapy was not due to a synergistic effect, but rather a result of reducing both antibiotic-sensitive and -resistant strains of bacteria as well as its ability to prevent selection of antibiotic-resistant variants on the skin of acne patients maintaining the efficacy of topical antibiotics. ${ }^{28,30}$

The mechanism of action of the combination of topical antibiotics and BP is likely multifactorial. The combination of keratolytic, bactericidal, and anti-inflammatory properties of $\mathrm{BP}$ and the antibacterial and anti-inflammatory properties of topical antibiotics make this treatment useful for both inflammatory and noninflammatory acne.

\section{Clindamycin I.2\% and benzoyl peroxide 5\%}

Similar to erythromycin, several studies involving topical combinations of clindamycin and BP demonstrated efficacy in treating acne vulgaris and have been found to be superior to monotherapy with either medication alone. ${ }^{4-7,31,32} \operatorname{In} 1997$, Lookingbill et a $\mathrm{l}^{32}$ performed two double-blind controlled clinical trials to determine the efficacy and safety of a combination clindamycin $1.2 \%$ and BP 5\% gel (clindamycin-BP $5 \%)$ compared to either agent alone and vehicle. Efficacy and safety were evaluated at baseline and again at weeks 2, 5, 8, and 11. Efficacy was evaluated using inflammatory and noninflammatory lesion counts, and safety was evaluated using scores for facial erythema, peeling, burning, dryness, and pruritus. At week 11, clindamycin-BP 5\% had a $66 \%$ mean reduction in inflammatory and noninflammatory lesions compared to $41 \%$ reduction in BP-treated patients, $36 \%$ reduction in clindamycin-treated patients, and $10 \%$ reduction in those patients treated with the vehicle. Clindamycin-BP $5 \%$ also had a statistically significant improvement in global improvement scores. Overall, combination therapy was fairly well tolerated, but was associated with mild erythema, peeling, and irritation. The rate of these adverse events was comparable to BP alone. ${ }^{32}$

\section{Clindamycin I.2\% and benzoyl peroxide $2.5 \%$}

Because of concerns for decreased compliance, fixed combinations of clindamycin and BP $2.5 \%$ have been developed to decrease the side effects associated with higher concentrations of BP. The major concern regarding reducing or changing the concentration of $\mathrm{BP}$ was the possibility that lower concentrations may be associated with reduced efficacy. However, several studies have demonstrated the clinical efficacy of clindamycin-BP $2.5 \%$ for the treatment of acne vulgaris, indicating that it may be just as effective as $5 \%$ and $10 \%$ concentrations for the treatment of acne vulgaris. ${ }^{4,5,31,33}$ Additionally, in an in vitro penetration study, Bucks et a ${ }^{34}$ found that reducing the BP concentration from $5 \%$ to $2.5 \%$ still maintained a similar amount of total BP delivered to the 
skin, while also leading to a $33 \%$ reduction in local irritation, thus increasing patient compliance.

A meta-analysis by Seidler and Kimball ${ }^{3}$ examined the efficacy of fixed combination treatments containing clindamycin-BP $5 \%$ vs clindamycin-BP $2.5 \%$. A Cochrane review identified 126 randomized controlled trials in which subjects were treated with BP $2.5 \%$ or $5 \%$ and $1 \%$ or $1.2 \%$ clindamycin. A total of 5,737 patients were included. Of them, 1,069 patients were in the clindamycin-BP 5\% group and 872 patients were in the clindamycin-BP $2.5 \%$ group. The remainder of the patients received either BP alone, clindamycin alone, or vehicle. They found that the combination of clindamycin and BP in any concentration was superior to either single agent alone in treating inflammatory lesions. Clindamycin-BP $2.5 \%$ was comparable to clindamycin-BP $5 \%$ in treating acne vulgaris. Interestingly, clindamycin-BP $2.5 \%$ led to a significantly greater absolute reduction in lesion count for both inflammatory and noninflammatory lesions compared to clindamycin-BP 5\%. ${ }^{3}$ Weighted absolute reductions in inflammatory and noninflammatory lesion counts for clindamycin-BP 5\% were 14.81 and 16.83 , respectively. Weighted absolute reduction in inflammatory and noninflammatory lesion counts for clindamycin-BP $2.5 \%$ were 25.37 and 20.83, respectively. It is unclear whether this advantage is due to increased efficacy or increased compliance due to decreased localized irritation from a lower concentration of BP. However, this combination is an effective treatment for mild-to-moderate acne vulgaris. Despite the various combinations of clindamycin and $\mathrm{BP}$ available, the concentration of BP for optimal efficacy and tolerability is still unknown.

\section{Clindamycin phosphate I.2\% and benzoyl peroxide $3.75 \%$}

Recently, a clindamycin phosphate $1.2 \%$ and BP $3.75 \%$ fixed combination aqueous gel was created as a novel intervention for the treatment of moderate-to-severe acne vulgaris. At BP $3.75 \%$, the combination was designed to have improved efficacy over the clindamycin-BP $2.5 \%$, with increased tolerability over the clindamycin-BP $5 \%$ combination. Pariser et a ${ }^{35}$ evaluated the safety and efficacy of clindamycin-BP 3.75\% aqueous gel in a study involving 498 patients with moderateto-severe acne. Patients with moderate-to-severe acne were randomized to clindamycin-BP $3.75 \%$ or vehicle.

\section{Results}

After 12 weeks of treatment, Pariser et $\mathrm{al}^{35}$ found that clindamycin-BP $3.75 \%$ was statistically superior to vehicle for the treatment of moderate-to-severe acne vulgaris. The mean percentage reduction in lesion counts was significantly higher in the clindamycin-BP $3.75 \%$ group compared to vehicle. The mean percentage change from baseline to week 12 for inflammatory and noninflammatory lesion counts on clindamycin-BP $3.75 \%$ was $60.6 \%$ and $51.6 \%$, respectively. The mean percentage change from baseline to week 12 for inflammatory and noninflammatory lesion counts on vehicle was $31.4 \%$ and $27.4 \%$, respectively. Treatment success, defined as at least a 2-grade improvement in Evaluator Global Severity Score, occurred in $34.3 \%$ vs $15.6 \%$ in patients treated with clindamycin-BP $3.75 \%$ compared to patients treated with vehicle by the end of the study.

Subjective assessment of efficacy included assessment of subject self-assessment (SSA), patient satisfaction survey (PSS), degree of oiliness and shininess, and acne-specific quality of life questionnaires.

At the end of the study, an SSA of "clear" or "almost clear" was reported in $34.9 \%$ of cases in the clindamycin-BP $3.75 \%$ group compared to $17.4 \%$ in the vehicle group. More importantly, over $96 \%$ of patients reported some improvement in their acne on SSA following 12 weeks of treatment. Mean PSS scores at the end of the study increased from baseline by $56 \%$ in the clindamycin-BP $3.75 \%$ group compared to $41 \%$ in the vehicle group. Additionally, the percent of patients reporting "none" to "mild" oiliness and shininess increased by week 12 by $22.6 \%$ vs $15.6 \%$ for clindamycin-BP $3.75 \%$ and vehicle, respectively. This corresponded with a decrease in patients reporting "moderate" to "severe" oiliness or shininess. Interestingly, the results of the acne quality of life assessment did not show a meaningful difference between the two groups at the end of the study.

\section{Limitations}

Despite the improvement associated with clindamycin-BP $3.75 \%$ gel, this trial only evaluated this fixed combination gel to the vehicle. Therefore, it is impossible to determine whether it is superior or inferior to the existing $2.5 \%$ or $5 \%$ combination therapies. In this study, mean improvement after 12 weeks of treatment with clindamycin-BP $3.75 \%$ or vehicle was $60.6 \%$ vs $31.4 \%$ in inflammatory lesions and $51.6 \%$ vs $27.4 \%$ in noninflammatory lesions, respectively. ${ }^{35}$ In a similar study using clindamycin-BP $2.5 \%$, the reported reduction was $64.1 \%$ vs $34.3 \%$ in inflammatory lesions and $48.7 \%$ vs $26.0 \%$ in noninflammatory lesions with clindamycin-BP $2.5 \%$ and vehicle, respectively. ${ }^{31}$ Comparative effectiveness studies of the BP-clindamycin combination therapies have not been performed. Further studies evaluating clindamycin-BP $3.75 \%$ efficacy and tolerability compared 
to clindamycin monotherapy, BP monotherapy, and other fixed combination medications could be useful.

\section{Clindamycin phosphate $1.2 \%$ and benzoyl peroxide $3.75 \%$ in women}

Unlike males who typically outgrow acne with time, a subset of female patients may experience acne into adulthood, and this may include as much as half of all adult females between the ages of 20-29 years. ${ }^{36,37}$ In this subset of patients, hormonal dysregularities such as polycystic ovarian syndrome and hyperandrogenism are more common. ${ }^{36}$ For this reason, these patients tend to not respond as robustly to standard acne treatments, and gynecological follow-up is often required. It is generally accepted that acne in adult females typically presents as mild-to-moderate inflammatory acne that is usually limited to the jawline, chin, and neck, otherwise known as the "U-zone". ${ }^{36,37}$ However, in a recent large-scale international study, researchers found that almost $90 \%$ of women with acne did not have it limited to the "U-zone", and a mixed inflammatory and noninflammatory acne was the most common presentation. ${ }^{38}$ These results suggested that the presentation of facial acne in adult females might be more similar to adolescent acne than once believed.

A subgroup analysis performed by Pariser et $\mathrm{al}^{35}$ in their study suggested a slightly greater efficacy for clindamycin-BP $3.75 \%$ in female patients compared to males. The mean percentage reduction of noninflammatory lesions at the end of the study was $55.7 \%$ vs $48.1 \%$ in female and male patients, respectively. The mean percentage reduction of inflammatory lesions at the end of the study was $65.3 \%$ vs $55.8 \%$ in female and male patients, respectively. ${ }^{35}$ Although this information suggests that the response of male and female patients to clindamycin-BP $3.75 \%$ may differ, it is unclear whether there is a distinction between acne in different sexes or different age groups. Additionally, the female patients included in this analysis were not limited to those with "U-zone" distributed acne. Further clinical trials are necessary to determine whether clindamycin-BP $3.75 \%$ is more effective in female patients and whether the pathogenesis of acne in this subset of patients is different from other patients.

\section{Conclusion}

Acne vulgaris is the most common skin disease worldwide and is a frequent source of morbidity for affected patients. A significant proportion of treatment options exist for acne vulgaris, and combination therapy is commonly employed to combat the various causes of acne pathogenesis. Clindamycin in conjunction with BP has become part of the mainstay of treatment for patients with inflammatory and noninflammatory acne. However, the optimal combination of efficacy and tolerability has yet to be determined. Pariser et $\mathrm{al}^{35}$ demonstrated that clindamycin-BP $3.75 \%$ is safe and effective for the treatment of acne vulgaris, suggesting that this formulation may be an effective treatment regimen for affected patients. It is clear that this specific combination has the ability to reduce both inflammatory and noninflammatory acne lesions and to be well tolerated. Further studies would be useful to assess comparative effectiveness to other $\mathrm{BP}-$ clindamycin products and monotherapy antibiotics and BP.

\section{Acknowledgment}

No external funding was secured for this study.

\section{Disclosure}

The authors report no conflicts of interest in this work.

\section{References}

1. Acne [webpage on the Internet]. American Academy of Dermatology; 2015. Available from: http://www.aad.org/media-resources/stats-andfacts/conditions/acne. Accessed April 21, 2015.

2. Thiboutot D, Gollnick H, Bettoli V, et al. New insights into the management of acne: an update from the Global Alliance to Improve Outcomes in Acne group. J Am Acad Dermatol. 2009;60:S1-S50.

3. Seidler EM, Kimball AB. Meta-analysis of randomized controlled trials using $5 \%$ benzoyl peroxide and clindamycin versus $2.5 \%$ benzoyl peroxide and clindamycin topical treatments in acne. JAm Acad Dermatol. 2011;65:e117-e119.

4. Thiboutot D, Zaenglein A, Weiss J, Webster G, Calvarese B, Chen D. An aqueous gel fixed combination of clindamycin phosphate $1.2 \%$ and benzoyl peroxide $2.5 \%$ for the once-daily treatment of moderate to severe acne vulgaris: assessment of efficacy and safety in 2813 patients. J Am Acad Dermatol. 2008;59:792-800.

5. Webster G, Rich P, Gold MH, Mraz S, Calvarese B, Chen D. Efficacy and tolerability of a fixed combination of clindamycin phosphate (1.2\%) and low concentration benzoyl peroxide (2.5\%) aqueous gel in moderate or severe acne subpopulations. J Drugs Dermatol. 2009;8:736-743.

6. Green L, Cirigliano M, Gwazdauskas JA, Gonzalez P. The tolerability profile of clindamycin $1 \%$ /benzoyl peroxide $5 \%$ gel vs adapalene $0.1 \%$ / benzoyl peroxide $2.5 \%$ gel for facial acne: results of two randomized, single-blind, split-face studies. J Clin Aesthet Dermatol. 2012;5: $16-24$.

7. Guerra-Tapia A. Effects of benzoyl peroxide $5 \%$ clindamycin combination gel versus adapalene $0.1 \%$ on quality of life in patients with mild to moderate acne vulgaris: a randomized single-blind study. J Drugs Dermatol. 2012;11:714-722.

8. Feldman SR, Chen DM. How patients experience and manage dryness and irritation from acne treatment. J Drugs Dermatol. 2011;10: 605-608.

9. Dalgard F, Gieler U, Holm J, Bjertness E, Hauser S. Self-esteem and body satisfaction among late adolescents with acne: results from a population survey. J Am Acad Dermatol. 2008;59:746-751.

10. Oberemok SS, Shalita AR. Acne vulgaris, I: pathogenesis and diagnosis. Cutis. 2002;70:101-105.

11. Jahns AC, Eilers H, Ganceviciene R, Alexeyev OA. Propionibacterium species and follicular keratinocyte activation in acneic and normal skin. Br J Dermatol. 2015;172:981-987. 
12. Thiboutot DM, Knaggs H, Gilliland K, Hagari S. Activity of type 1 5 alpha-reductase is greater in the follicular infrainfundibulum compared with the epidermis. Br J Dermatol. 1997;136:166-171.

13. Guy R, Ridden C, Kealey T. The improved organ maintenance of the human sebaceous gland: modeling in vitro the effects of epidermal growth factor, androgens, estrogens, 13-cis retinoic acid, and phenol red. J Invest Dermatol. 1996;106:454-460.

14. Kurokawa I, Umeda K, Nishimura K, et al. Filaggrin expression and the pathogenesis of epidermal cysts. Br J Dermatol. 2007;157:415-416.

15. Akamatsu H, Zouboulis CC, Orfanos CE. Control of human sebocyte proliferation in vitro by testosterone and 5-alpha-dihydrotestosterone is dependent on the localization of the sebaceous glands. J Invest Dermatol. 1992;99:509-511.

16. Ottaviani M, Alestas T, Flori E, Mastrofrancesco A, Zouboulis CC, Picardo M. Peroxidated squalene induces the production of inflammatory mediators in $\mathrm{HaCaT}$ keratinocytes: a possible role in acne vulgaris. J Invest Dermatol. 2006;126:2430-2437.

17. Harvey A, Huynh TT. Inflammation and acne: putting the pieces together. J Drugs Dermatol. 2014;13:459-463.

18. Nagy I, Pivarcsi A, Koreck A, Széll M, Urbán E, Kemény L. Distinct strains of Propionibacterium acnes induce selective human betadefensin-2 and interleukin- 8 expression in human keratinocytes through toll-like receptors. J Invest Dermatol. 2005;124:931-938.

19. Holland DB, Jeremy AH. The role of inflammation in the pathogenesis of acne and acne scarring. Semin Cutan Med Surg. 2005;24:79-83.

20. Seidler EM, Kimball AB. Meta-analysis comparing efficacy of benzoyl peroxide, clindamycin, benzoyl peroxide with salicylic acid, and combination benzoyl peroxide/clindamycin in acne. J Am Acad Dermatol. 2010;63:52-62.

21. Waller JM, Dreher F, Behnam S, et al. 'Keratolytic' properties of benzoyl peroxide and retinoic acid resemble salicylic acid in man. Skin Pharmacol Physiol. 2006;19:283-289.

22. Hegemann L, Toso SM, Kitay K, Webster GF. Anti-inflammatory actions of benzoyl peroxide: effects on the generation of reactive oxygen species by leucocytes and the activity of protein kinase $\mathrm{C}$ and calmodulin. Br J Dermatol. 1994;130:569-575.

23. Leyden JJ, Wortzman M, Baldwin EK. Antibiotic-resistant Propionibacterium acnes suppressed by a benzoyl peroxide cleanser $6 \%$. Cutis. 2008;82:417-421

24. Mohd Nor NH, Aziz Z. A systematic review of benzoyl peroxide for acne vulgaris. J Dermatolog Treat. 2013;24:377-386.

25. Leccia MT, Auffret N, Poli F, Claudel JP, Corvec S, Dreno B. Topical acne treatments in Europe and the issue of antimicrobial resistance. J Eur Acad Dermatol Venereol. 2015;29:1485-1492.
26. Del Rosso JQ, Schmidt NF. A review of the anti-inflammatory properties of clindamycin in the treatment of acne vulgaris. Cutis. 2010;85:15-24.

27. Leyden JJ, Del Rosso JQ, Webster GF. Clinical considerations in the treatment of acne vulgaris and other inflammatory skin disorders: focus on antibiotic resistance. Cutis. 2007;79:9-25.

28. Eady EA, Farmery MR, Ross JI, Cove JH, Cunliffe WJ. Effects of benzoyl peroxide and erythromycin alone and in combination against antibiotic-sensitive and -resistant skin bacteria from acne patients. Br J Dermatol. 1994;131:331-336.

29. Chalker DK, Shalita A, Smith JG, Swann RW. A double-blind study of the effectiveness of a $3 \%$ erythromycin and 5\% benzoyl peroxide combination in the treatment of acne vulgaris. J Am Acad Dermatol. 1983;9:933-936.

30. Harkaway KS, McGinley KJ, Foglia AN, et al. Antibiotic resistance patterns in coagulase-negative staphylococci after treatment with topical erythromycin, benzoyl peroxide, and combination therapy. Br J Dermatol. 1992;126:586-590.

31. Gold MH. A new, once-daily, optimized, fixed combination of clindamycin phosphate $1.2 \%$ and low-concentration benzoyl peroxide $2.5 \%$ gel for the treatment of moderate-to-severe acne. J Clin Aesthet Dermatol. 2009;2:44-48

32. Lookingbill DP, Chalker DK, Lindholm JS, et al. Treatment of acne with a combination clindamycin/benzoyl peroxide gel compared with clindamycin gel, benzoyl peroxide gel and vehicle gel: combined results of two double-blind investigations. J Am Acad Dermatol. 1997;37:590-595.

33. Mills OH, Kligman AM, Pochi P, Comite H. Comparing 2.5\%, 5\%, and $10 \%$ benzoyl peroxide on inflammatory acne vulgaris. Int J Dermatol. 1986;25:664-667.

34. Bucks D, Sarpotdar P, Yu K, Angel A, Del Rosso J. The development and optimization of a fixed combination of clindamycin and benzoyl peroxide aqueous gel. J Drugs Dermatol. 2009;8:634-638.

35. Pariser DM, Rich P, Cook-Bolden FE, Korotzer A. An aqueous gel fixed combination of clindamycin phosphate $1.2 \%$ and benzoyl peroxide $3.75 \%$ for the once-daily treatment of moderate to severe acne vulgaris. J Drugs Dermatol. 2014;13:1083-1089.

36. Holzmann R, Shakery K. Postadolescent acne in females. Skin Pharmacol Physiol 2014;27(Suppl 1):3-8.

37. Preneau S, Dreno B. Female acne - a different subtype of teenager acne? J Eur Acad Dermatol Venereol. 2012;26:277-282.

38. Dréno B, Thiboutot D, Layton AM, et al. Large-scale international study enhances understanding of an emerging acne population: adult females. J Eur Acad Dermatol Venereol. 2015;29:1096-1106.
Clinical, Cosmetic and Investigational Dermatology

\section{Publish your work in this journal}

Clinical, Cosmetic and Investigational Dermatology is an international, peer-reviewed, open access, online journal that focuses on the latest clinical and experimental research in all aspects of skin disease and cosmetic interventions. All areas of dermatology will be covered; contributions will be welcomed from all clinicians and

\section{Dovepress}

basic science researchers globally. This journal is indexed on CAS The manuscript management system is completely online and includes a very quick and fair peer-review system, which is all easy to use. Visit http://www.dovepress.com/testimonials.php to read real quotes from published authors. 